\title{
The SICSA Workshop Series on Technologies for Health and Wellness (THAW)
}

\author{
Marilyn McGee Lennon \\ Mobiquitous Research Lab \\ Department of Computer \\ and Information Sciences \\ University of Strathclyde \\ marilyn.lennon@strath.ac.uk
}

\author{
Lynne Baillie \\ ITT Research Group \\ Glasgow Caledonian University \\ l.baillie@gcu.ac.uk
}

\begin{abstract}
This paper provides a brief overview of the Technologies for Health and Wellness (THAW) workshop series. These have been funded by the Scottish Informatics and Computing Science Alliance (SICSA).
\end{abstract}

Technology for health and wellness, healthcare, wellbeing, mhealth, ehealth, telehealh.

\section{MOTIVATION AND BACKGROUND}

The Scottish Informatics and Computer Science Alliance (SICSA) is a collaboration of Scottish Universities whose goal is to develop and extend Scotland's position as a world leader in Informatics and Computer Science research and education.

SICSA's 4 main research themes are:

1. Complex Systems Engineering;

2. Human Computer Interaction;

3. Next Generation Internet;

4. Modelling and Abstraction.

Each theme delivers a schedule of meetings, seminars, workshops and master-classes throughout the year. Events are held across Scotland and are open to all Informatics and Computing Science academics, lecturing staff and $\mathrm{PhD}$ researchers working within Scotland. Events are funded by SICSA and they are usually completely free to attend.

In the past decade there has been a significant increase in the interest in digital health within the multimodal and Human Computer Interaction $(\mathrm{HCl})$ research communities within SICSA. In addition to this, there has been increasing outside interest and potential for collaboration with emerging innovation centres, the NHS, charities and carer groups and other related research work streams relating to health, wellness, citizens and cities within SICSA. In 2012 Lynne Baillie and Marilyn Lennon argued for, and received funding for a 'Technology for Health and Wellness' (THAW) subtheme as part of the Human Computer Interaction SICSA research theme. This has resulted in a growing network of researchers across SICSA in this area mainly through the series of THAW workshops that have been running across the SICSA institutions.

\section{DIGITAL HEALTH}

Due to unprecedented changes in demographics and public health patterns (i.e. ageing populations and the rising prevalence of multiple chronic and long-term health conditions), there is broad policy support for shifting from the current position of reactive health-care delivery in hospitals to proactive prevention and/or self -management within the community. Equally, social care agendas also seek to encourage people to live longer, healthier independent lives in their own homes. These significant societal changes raise new and important research challenges for a wide spectrum of disciplines within the scientific community: computer science, $\mathrm{HCl}$, health informatics, health services research, sociology for example. These challenges include developing, testing, piloting and rolling-out increasingly innovative technology around the home, assessing the ease of use, usefulness, usability and 'fitness-for-purpose' of these new technologies and last but not least, measuring their impact on health, wellbeing and lifestyle (e.g. engagement, immersion, motivation, persuasion, visualisation, self -management, behaviour modification, gamification).

The challenge and complexity of the tasks involved requires without doubt a multidisciplinary approach to understanding the issues at stake and designing, piloting, implementing and evaluating appropriate solutions. We believe this requires sharing information, knowledge and experience between researchers from a wide range of disciplines with those designing, delivering and commissioning new products and services within health and social care. 


\section{TECHNOLOGY FOR HEALTH AND WELLNESS (THAW) WORKSHOP SERIES}

The THAW workshops held so far include:

Workshop 1 - Oct. 2011, Glasgow Caledonian University: The Grand Challenges for Health and Wellness Technology for the Home. Theme: raising issues, outlining pathways and discussing concerns.

Workshop 2 - March 2014, Glasgow Caledonian University. Sharing findings from current and recent projects undertaken in the field e.g. $\mathrm{MATCH}$, Envisage, MultiMemoHome, COBALT and Haven.

The workshop also discussed funding and possible collaboration on a Scotland and EU level and also how to take forward the key research agendas in this area (informed by workshop 1)

Workshop 3 - June 2014, (University of Strathclyde). Aim: To extend to the network of researchers. Also to meet and discuss the launch of the Digital Health Institute of Scotland with key people across our now established network.

Workshop 4 - Sept. 2014, (BCS Health Informatics Scotland Conference). Aim to further extend the network. We were invited to hold this workshop as part of the annual BCS Health Informatics Scotland Conference ${ }^{1}$ to highlight the existence of the THAW workshop theme within SICSA to a wider audience.

Workshop 5 - Nov. 2014, (University of Glasgow). We were invited by the Scottish Centre for Telehealth and Telecare to run our fifth THAW workshop as part of the National Digital Health Care week. The aim of this workshop was: To highlight the innovation across SICSA institutions in the area of digital health and to think about how we can work more closely with NHS, the private and third sectors to bring our innovation closer to market ready products and into mainstream routine care pathways.

\section{THE FUTURE FOR THE THAW WORKSHOP SERIES}

THAW has proved a popular workshop series in the UK and this has allowed researchers from computing science, heath and informatics to come together to share and discuss state of the art in technologies for health and wellness and also to form new collaborations within and across disciplines and to unite experts to collectively shape and drive the future research agenda for technologies for health and wellness.

\footnotetext{
${ }^{1}$ http://www.knowledge.scot.nhs.uk/his/events/healthinformatics-scotland-conference-2014.aspx
}

In April 2015, in Seoul, South Korea some of the THAW workshop team, together with a world leading international committee will run a workshop at the world's top Human Computer Interaction conference - ACM CHI 2015.

The aim of this particular workshop - "Crossing $\mathrm{HCl}$ and Health: Advancing health and wellness technology research in home and community settings" - is to get academics, industry practitioners and service providers crossing both the health and $\mathrm{HCl}$ fields to share expertise and experience regarding how new health technologies products and ideas can be designed, developed, implemented and evaluated in order to complete the journey from 'the lab' to mainstream service delivery for health and wellness technology. The workshop will bring together world renowned experts in this area and to explore the challenges for advancing this field (evaluating at scale, ethics, adapting funding models) and also the opportunities that lie ahead.

We invite short papers and case studies from people working in the cross-over between health and $\mathrm{HCl}$ in a non-clinical setting (technology for the home and community setting rather than hospital or GP based systems). We would like papers to highlight what the grand challenges in this area are from utilizing existing and future technology concepts to recruiting users and patients to designing with and for diverse stakeholders to methodologies for evaluating in homes, communities and at scale.

The goal of the workshop is to share experiences and to identify not only the challenges of working between disciplines but also the opportunities that arise because of the crossing over between health and $\mathrm{HCl}$ research in recent years. This will be a full one day workshop on Saturday $18^{\text {th }}$ April and will combine short oral presentations for the accepted authors with posters, demos, discussions and interactive sessions to actively prepare a vision statement for $\mathrm{HCl}$ and Health research during the workshop that can be shared with the rest of the $\mathrm{CHI}$ community.

The outputs from the workshop will be a paper on addressing questions such as: Are we ready for digitally enabled self-care? How can we continue to advance research that crosses methodological and cultural boundaries between Health and $\mathrm{HCl}$ ? 